\title{
ABBREVIATIONS
}

ACIO

Ael.

Aesch.

Aeschin.

$A J P h$

André Pont.

André $T r$.

Anth. Pal.

Apollod.

App. BC

Ap. Rhod.

Atti del Convegno Intemazionale Ovidiano, Sulmona, maggio 1959, Rome, 1959

Claudius Aelianus (2nd-3rd cent. AD), rhetorician and moralist
$N A$
De Natura Animalium

Aeschylus, son of Euphorion ( $525 / 4-456$ BC), Greek tragedian

Ag.

Choeph.

Mymid.

Sept.
Agamemnon

Choephori

Mymidones

Septem contra Thebas

(Seven against Thebes)

Aeschines (c.397-c. $322 \mathrm{BC})$, Athenian orator American Journal of Philology

J. M. André, Ovide: Pontiques, Paris, 1977

J. M. André, Ovide: Tristes, Paris, 1968

Anthologia Palatina, the 'Greek Anthology', a late roth-cent.-AD collection of epigrams, based on earlier anthologies, e.g. that of Meleager of Gadara (fl. c. 100 BC.)

Apollodorus, Greek mythographer of ?Antonine period, author of Bibliotheca, compendium of myths

Epit.

Epitome

Appianus of Alexandria (2nd cent. AD): Roman-naturalized Greek historian and procurator Augusti: the Bellum Civile = Books I 3-I 7 of his Romaika

Apollonius Rhodius, Hellenistic (3rd cent. BC) Greek epic poet and scholar 
Apul.Apol.

Arat. Phaen.

Aricescu

Arist.

Athen.

Aug. Res Gest.

Aul. Gell. NA

Bakker

Benedum

BICS

Burman

$C A$

Caes. $B C$

Callim.

Apuleius Madaurensis (2nd cent. AD), African-Roman writer and rhetorician: his Apologia is also known as Pro se de magia.

Aratus of Soli (c. 3 I s-240 BC), Greek Hellenistic poet, author of a Stoicized star-guide in verse, the Phaenomena

A. Aricescu, 'Le mur d'enceinte de Tomi à l'époque d'Ovide', Ovidianum, pp. 85-90

Aristoteles (Aristotle) of Stagira (384-322 BC), Greek philosopher

$\begin{array}{ll}H A & \text { Historia Animalium } \\ \text { Poet. } & \text { Poetica }\end{array}$

Athenaeus of Naucratis (fl. C. AD 200), author of discursive treatise on food (and literary dinner-table chat), the Deipnosophistae

Augustus (63 BC-AD 14), first emperor of Rome, in his Res Gestae wrote a (carefully slanted) record of his achievements Aulus Gellius, (c. AD I 30-c. AD I 80), Roman writer, author of Noctes Atticae

J. T. Bakker, Publ. Ovid. Nasonis Tristium Li $V$, Groningen, 1946

Jost Benedum, Studien zur Dichtkunst des späten Ovids, Giessen, 1967

Bulletin of the Institute of Classical Studies of the University of London

P. Burman, Publii Ovidii Nasonis Tristium Lib. IV Ex Ponto Lib. IV . . Tom . . III. Pars II, Amsterdam, 1727

\section{Classical Antiquity}

C. Julius Caesar (100-44 BC), Roman general and Dictator: wrote the $D e$ Bello Civili to justify his part in the civil wars

Callimachus of Cyrene (c. 305 c. 240 BC), Alexandrian scholar-poet 


$\begin{array}{ll}\text { Epigr. } & \text { Epigrammata } \\ \text { H. } & \text { Hymni }\end{array}$

Catull.

Cels.

Cic.

CIL

CJ

Claassen $O P P$

Claassen PEV

Colakis

CQ

Davisson DCE

Davisson $M P O$
C. Valerius Catullus (?84-?54 BC), Roman elegiac and lyric poet

A. Cornelius Celsus (Ist cent. AD), Roman encyclopaedist

M. Tullius Cicero (ro6-43 BC), Roman writer, orator and statesman

Att.

Epistulae ad Atticum

Brut.

Brutus

De Orat.

De Oratore

Fam.

Epistulae ad Familiares

ND

De Natura Deorum

Phil.

Philippicae

Rep.

De Republica

Tusc. (Disp.)

Tusculanae

Disputationes

Corpus Inscriptionum Latinarum, Berlin, I863-

Classical Journal

J.-M. Claassen, 'Ovid's poetic Pontus', Papers of the Leeds Intemational Latin Seminar, vol. vi (I990), pp. 65-94

J.-M. Claassen, 'Poeta, Exsul, Vates: A stylistic and literary analysis of Ovid's Tristia and Epistulae ex Ponto', D.Litt. dissertation, Univ. of Stellenbosch, 1986

M. Colakis, 'Ovid as praeceptor amoris in Epistulae ex Ponto 3.I', CJ 82 (1987), pp. 210-I5

Classical Quarterly

M. T. Davisson, 'Duritia and creativity in exile: Epistulae ex Ponto 4.10', CA I (1982), pp. 28-42

M. T. Davisson, 'Magna tibi imposita est 
Davisson SSO

De Jonge

Della Corte GSO

Della Corte $O P$

Della Corte $O T$

Della Corte, Fasce

Dickinson

Diggle

Dio Cass.

Diog. Laert.

Dion. Hal.

Drucker

DS nostris persona libellis: playwright and actor in Ovid's Epistulae ex Ponto 3.I', CJ 79 (1984), pp. 324-39

'Sed sum quam medico notior ipse mihi: Ovid's use of some conventions in the exile epistles', $C A 2$ (1983), pp. I7I-82

T. J. De Jonge, Publ. Ovid. Nasonis Tristium liber IV, Diss. Groningen, I95 I

F. Della Corte, 'Il Geticus semo di Ovidio', Scritti in onore di Giuliano Bonfante (Brescia, I975), i, pp. 205-I6

F. Della Corte, Ovidio: I Pontica, Genoa, 1974

F. Della Corte, Ovidio: I Tristia, Genoa, I973

F. Della Corte, S. Fasce, Ovidius Opere, II: Tristia, Ibis, Ex Ponto, Halieuticon liber, Turin, I 986 [not seen by me]

R. J. Dickinson, 'The Tristia: poetry in exile', in Ovid (ed. J. W. Binns, London, I973), pp. I 54-90

J. Diggle, 'Notes on Ovid's Tristia, Books I-II', CQ 30 (1980), pp. 40I-I9

Cassius Dio Cocceianus of Nicaea (2nd-3rd cent. AD), Roman statesman and historian

Diogenes Laertius (? early 3rd cent. AD), Greek philosophical writer and biographer

Dionysius Halicarnassensis (Ist cent. BC-Ist cent. AD), Rome-based Greek rhetorician and historian

$$
\text { Ant. Rom. Antiquitates Romanae }
$$

M. Drucker, Der verbannte Dichter und der Kaiser-Gott: Studien zu Ovids späten Elegien, Diss. Heidelberg, 1977

Diodorus Siculus ( $f$. Ist cent. BC), Greek historian 
Enc. Brit."

Enn.

Eur.

Euseb. Chron.

Evans $P C$

Evans $W W$

Fairweather

Florus

Focardi

Fränkel q.v.)
Encyclopaedia Britannica, I Ith ed., Cambridge/New York, 1910

Q. Ennius (239-169 BC), of Rudiae in Calabria, Roman epic poet Ann. Annales

Euripides $(c .485-c .406 \mathrm{BC})$, Athenian tragic playwright

$\begin{array}{ll}\text { Androm. } & \text { Andromache } \\ \text { Bacch. } & \text { Bacchae } \\ \text { Hel. } & \text { Helena } \\ \text { HF } & \text { Hercules Furens } \\ \text { Med. } & \text { Medea } \\ \text { Orest. } & \text { Orestes } \\ \text { Phoen. } & \text { Phoenissae }\end{array}$

Eusebi Chroniconum canonum quae supersunt, ed. A. Schoene, 2 vols., I866, I875, repr. Dublin/Zürich, 1967

H. B. Evans, Publica Camina: Ovid's Books from Exile, Univ. of Nebraska Press, 1983

H. B. Evans, 'Winter and warfare in Ovid's Tomis (Tristia 3.10)', CJ 70 (1975), pp. $\mathrm{I} \rightarrow 9$

J. Fairweather, 'Ovid's autobiographical poem, Tristia 4.10', CQ 37 (1987), pp. I $8 \mathrm{I}-96$

Publius Annius Florus (late Ist to early 2nd cent. AD), Roman historian and poet

G. Focardi, 'Difesa, preghiera, ironia nel II libro dei Tristia di Ovidio', SIFC 47 (1975), pp. 86-I 29

H. Fränkel, Ovid: A Poet between Two Worlds (Sather Classical Lectures, vol. 18), Univ. of California Press, 1945

Fredericks (= Nagle, 'Tristia 4.Io: Poet's autobiography and poetic autobiography', TAPhA 106 (1976), pp. $139-54$ 
Froesch OEP

Froesch $O D E$

Galasso (1987)

GER

Goold (see also

Wheeler-Goold)

Green $A A$

Green $A M$

Green $C B$

Green OEP

Green $S P$

Hall

Hdt.

Helzle (I)

Helzle (2)
H. Froesch, 'Ovids Epistulae ex Ponto IIII als Gedichtsammlung', Diss. Bonn, 1968 H. Froesch, Ovid als Dichter des Exils, Bonn, 1976

L. Galasso, 'Modelli tragici e ricodificazione elegiaca. Appunti sulla poesia ovidiana dell'esilio', $M D$ I 8 (1987), pp. 83-99

Greece and Rome

G. P. Goold, 'The cause of Ovid's exile', Illinois Class. Stud. 8 (1983), pp. 94-107

Peter Green, Alexander to Actium: The Historical Evolution of the Hellenistic Age, Berkeley/London, rev. ed. 1993.

Peter Green, Alexander of Macedon, 356-323 $B C$ : A Historical Biography, Harmondsworth, 1974, repr. Univ. of California Press, 199I

Peter Green, Classical Bearings: Interpreting Ancient History and Culture, London/New York, 1989

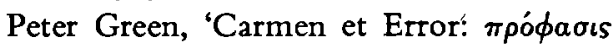
and airia in the matter of Ovid's exile', $C A 2$ (1982), pp. 202-20

Peter Green, Ovid: The Erotic Poems, Harmondsworth, 1982

Peter Green, The Shadow of the Parthenon:

Studies in Ancient History and Literature, Univ. of California Press, 1972

J. B. Hall, P. Ovidi: Nasonis Tristia. Stuttgart \& Leipzig (Teubner) 1995 .

Herodotus Halicarnassensis (c. $485-c .425 \mathrm{BC})$, Greek historian

M. Helzle, 'Mr and Mrs Ovid', G\&R 36 (1989), pp. I83-93

M. Helzle, 'Ovid's poetics of exile', Illinois Class. Stud. I 3 (1988), pp. 73-83 
Helzle (3)

Herescu

Herrmann

Hes.

$H H$

Hom.

Hor.

Housman $C P$

Hyg.

Isocr.

Juv.
M. Helzle, Publii Ovidii Nasonis Epistularum ex Ponto liber IV: A Commentary on Poems 1-7 and 16, Hildesheim, 1989

N. I. Herescu, 'Ovide, le Gétique (Pont. IV.13.I8: paene poeta Getes)', $A C I O$ vol. i, pp. 55-80 [cf. Orpheus 7 (1960), pp. I-26]

K. Herrmann, De Ovidii Tristium libris $V$, Diss. Leipzig, 1924

Hesiodus ( $f$. 8th -7 th cent. BC), early Greek didactic poet

Theog.

Theogonia

$W D$ Works \& Days (Opera et Dies)

Hymni Homerici

Homerus ( $f$. 8th cent. BC), Greek epic poet Il. Ilias

Od.

Odyssea

Q. Horatius Flaccus $(65-8$ BC), Roman lyric poet and satirist
$A P$
Ars Poetica
Carm. Saec.
Carmen Saeculare
Ep.
Epistulae
Odes (Carm.)
Odes (Carmina)
Sat.
Saturae, Sermones

J. Diggle \& F. R. D. Goodyear, The Classical Papers of A. E. Housman, vol. iii, 19151936, Cambridge, 1972

Hyginus (?2nd-3rd cent. AD), Roman mythographer

Fab.

Fabulae

Isocrates $(436-338 \mathrm{BC})$, Athenian educationalist

D. Iunius Iuuenalis (c. AD $55-c$. I 30), Roman satirist

Sat.

Saturae 
Kenney ( 1 )

Kenney (2)

Kenney (3)

Labate

Laus. Pison.

Lee

Little

$\operatorname{Liv}(y)$

Lozovan $O B$

Lozovan $R P$

Lucias

Luck $\operatorname{Tr} . i$

Luck $T r . i i$

Lucr.
E. J. Kenney, 'The poetry of Ovid's exile', $P C P h S$ I I (1965), pp. 37-49

E. J. Kenney, 'Ovid', Cambridge History of Classical Literature, vol. ii, Cambridge, I982, pp. 420-57, esp. pp. 44Iff.

E. J. Kenney, art. 'Ovid' in $O C D^{2}$, pp. $763-5$

M. Labate, 'Elegia triste e elegia lieta: un caso di rinconversione letteraria', $M D$ I9 (I987), pp. 91-I 29

Laus Pisonis, Latin panegyric (?Ist cent. AD), author unknown

A. G. Lee, 'An appreciation of Tristia III.8', GER I 8 (1949), pp. I I 3-20

D. Little, 'Ovid's last poems: cry of pain from exile or literary frolic in Rome?', Prudentia 22 (1990), pp. 23-39

T. Livius Patavinus (?59 BC-?AD I7), Roman historian per. periochae

E. Lozovan, 'Ovide et le bilingualisme', Ovidiana, pp. 396-403

E. Lozovan, 'Réalités Pontiques et nécessités littéraires chez Ovide', $A C I O$ vol. ii, pp. $355-70$

Lucianus Sophista (b. c. AD I 20), of Samosata, Greek satirist

$\begin{array}{ll}\text { Adv. Indoct. } & \text { Adversus Indoctum } \\ \text { Tox. } & \text { Toxaris }\end{array}$

G. Luck, P. Ovidius Naso, Tristia, vol. i (Text), Heidelberg, 1967

G. Luck, P. Ovidius Naso, Tristia, vol. ii (Commentary), Heidelberg, 1977

T. Lucretius Carus (?94-?55 BC), Roman didactic poet

lxix 
Marchesi

Marg

Marshall

Mart.

Martini

$M D$

Meiser

Mnemos.

Nagle

Némethy $E P$

Némethy SC

Némethy $T r$.

Nep.

$O C D^{2}$

C. Marchesi, 'Il II libro ovidiano dei Tristia', Atene e Roma is (1912), pp. 159-67

W. Marg, 'Zur Behandlung des Augustus in den Tristien', $A C I O$ vol. ii, pp. 345-54

A. J. Marshall, 'Library resources and creative writing at Rome', Phoenix 30 (1976), pp. $252-64$

M. Valerius Martialis (c. AD 40-c.104), Spanish-born Roman epigrammatist

E. Martini, ' $\mathrm{Zu}$ Ovids und Kallimachos' Ibis', Phil. Woch. 52 (1932), pp. I IOI-8

Materiali e discussioni per l'analisi dei testi classici (Pisa)

R. Meiser, 'Ueber Ovids Begnadigungsgesuch (Trist. II)', SBAW (1907), pp. I7I205

Mnemosyne

B. R. Nagle, The Poetics of Exile: Program and Polemic in the Tristia and Epistulae ex Ponto of Ovid (Coll. Latomus, vol. 170), Brussels, 1980

G. Némethy, Commentarius exegeticus ad Ovidii Epistulas ex Ponto, Budapest, I9Is

G. Némethy, Supplementum commentariorum ad Ovidii Amores Tristia et Epistulas ex Ponto, Budapest, 1922

G. Némethy, Commentarius exegeticus ad Ovidii Tristia, Budapest, 1913

Cornelius Nepos (c. 99-c. 24 BC), Gallicborn Roman biographer

Them.

Themistocles

Oxford Classical Dictionary, and ed., Oxford, 1970 
$O L D$

Ovid

Ovidiana

Ovidianum

Owen Tr. I

Owen Tr. II

Owen Tr. III

Paneg. Mess.

Paus.

PCPhS

Pers. Sat.

Phaedr.

Phil. Woch

Pind.
Oxford Latin Dictionary, ed. P. G. W. Glare, Oxford, 1968-82

Publius Ovidius Naso (43 BC-AD 17/18), Roman poet and exile

$\begin{array}{ll}\text { AA } & \text { Ars Amatoria } \\ \text { Am. } & \text { Amores } \\ \text { EP } & \text { Epistulae ex Ponto } \\ \text { Fast. } & \text { Fasti } \\ \text { Her. } & \text { Heroides } \\ \text { Met. } & \text { Metamorphoses } \\ \text { Rem. } & \text { Remedia Amoris } \\ \text { Tr. } & \text { Tristia }\end{array}$

N. I. Herescu, ed., Ovidiana: Recherches sur Ovide, Paris, 1958

N. Barbu, E. Dobroiu, M. Nasta, eds., Ovidianum: Acta conventus omnium gentium ovidianis studiis fovendis, Bucharest, 1976

S. G. Owen, Tristia Book I, and rev. ed., Oxford, I 890

S. G. Owen, P. Ovidi Nasonis Tristium Liber Secundus, Oxford, 1924

S. G. Owen, Ovid: Tristia Book III, Oxford, I 889

Panegyricus ad Messallam

Pausanias Periegeta (?of Lydia), fl. AD I so, Greek travel-writer

Proceedings of the Cambridge Philological Society

Aulus Persius Flaccus (AD 34-62), Roman author of Saturae

Phaedrus (c. I5 BC-c. AD 50), freedman of Augustus, fabulist

Philologische Wochenschrift

Pindarus Lyricus (5I8-438 BC), Boeotian lyric poet

Isthm.

Isthmian Odes

Pyth. 
$P I R^{1}$

Plat.

Platner-Ashby

Plaut.

Plin. Ep.

Plin. $H N$

Plut.

Prosopographia Imperii Romani Saeculi I, II, $I I I$, Ist ed., ed. E. Klebs, H. Dessau, Berlin, I 897-8

Prosopographia Imperii Romani Saeculi I, II, $I I I$, 2nd ed., ed. E. Groag, A. Stein, Berlin \& Leipzig, 1933-

Plato, son of Ariston (c.429-347 BC), Athenian philosopher

$\begin{array}{ll}\text { Apol. } & \text { Apologia } \\ \text { Crit. } & \text { Crito } \\ \text { Phaedr. } & \text { Phaedrus } \\ \text { Rep. } & \text { Republic }\end{array}$

S. B. Platner \& T. Ashby, $A$ Topographical Dictionary of Ancient Rome, Oxford, 1929

Titus Maccius Plautus ( $f$. 3 rd cent. BC), Roman comic playwright

Rud.

Rudens

C. Plinius Caecilius Secundus (c. AD 6Ic.112), Roman lawyer and administrator, Epistulae (his public and literary correspondence)

C. Plinius Secundus (A D 23/4-79), uncle of the foregoing: administrator and scholar, published a Historia Naturalis in 37 books

Mestrius(?) Plutarchus of Chaeronea in Boeotia (c. AD 50-c. I20), Greek philosopher and biographer

Aem. Paul.

Ant.

Arist.

Caes.

Cat. Maj.

Lucull.

Marcell.

Mar.

Mor(al).
Aemilius Paullus Antonius Aristides Julius Caesar Cato Major Lucullus Marcellus Marius Moralia 


$\begin{array}{ll}\text { Pomp. } & \text { Pompeius } \\ \text { Rom. } & \text { Romulus } \\ \text { Sull. } & \text { Sulla } \\ \text { Them. } & \text { Themistocles } \\ \text { Timol. } & \text { Timoleon }\end{array}$

Priap.

Prop.

PWK

Res Gest.

Richmond

Richmond STP

$S B A W$

schol.

Scholte

Schubert

Sen. (I)

Sen. (2)
Priapea: collection of Augustan date: 'priapic' poems, one at least by Ovid

Sextus Propertius ( $54 / 47$ ?a. 2 BC), from Assisi in Umbria: Roman elegiac poet

Real-Encyclopädie d. klassischen Altertumswissenschaft, ed. A. Pauly, G. Wissowa, W. Kroll, K. Ziegler, Stuttgart-München, I 893-1972 [S-B = Supplement-Band]

See 'Aug. Res Gest.'

J. A. Richmond, P. Ovidi Nasonis Ex Ponto Libri Quattuor, Leipzig, 1990

J. A. Richmond, 'Some textual problems in Ovid's Ex Ponto', BICS Suppl. 5 (1988), pp. I I I-I 7

Sitzungsberichte der Bayerischen Akademie der Wissenschaften, Phil.-Hist. Klasse

scholium or scholiast

A. Scholte, Ex Ponto Liber I, herausgegeben und erklärt (Diss. Groningen), Amersfoort I 933

W. Schubert, 'Zu Ovid, Trist. 3.9', Gymnasium 97 (1990), pp. 154-64

L. Annaeus Seneca [the Elder] (c. 55 BC-c. AD 40), Spanish-born Roman historian and rhetorician

Controv. Controversiae

Suas.

Suasoriae

L. Annaeus Seneca [the Younger] (c. AD 
$\mathrm{I}-65$, son of the foregoing, Roman philosopher and tragedian
Benef.
De Beneficiis
Cons. ad Helv. Consolatio ad Helviam
Dial.
Dialogi
Epigr.
Epigrammata
Ep.
Epistulae

Serv.

M. Servius Honoratus (4th cent. $A D$ ), author of a Commentary on Virgil

Shackleton Bailey (I) D. R. Shackleton Bailey, 'Ovidiana', CQ 48 (1954), pp. I65-70

Shackleton Bailey (2) D. R. Shackleton Bailey, 'Notes on Ovid's poems from exile', CQ 32 (I982), pp. 39098

SIFC

Studi Italiani di Filologia Classica

Soph.

Sophocles, son of Sophilos (496-406 BC), Athenian tragedian

$\begin{array}{ll}\text { Aj. } & \text { Ajax } \\ \text { OT } & \text { Oedipus Tyrannus } \\ \text { Trach. } & \text { Trachiniae }\end{array}$

Staffhorst

U. Staffhorst, Publius Ovidius Naso: Epistulae ex Ponto III, 1-3 (Kommentar), Diss. Würzburg, 1965

Strabo

Strabo of Amaseia (64/3 BC-AD 25?), Stoic historian and geographer

Stud. Ovid.

F. Amaldi \& others, Studi Ovidiani, Rome, I959

Suda

Title of Greek encyclopedic lexicon (c. AD rooo): formerly supposed to have been written by 'Suidas'.

Suet.

C. Suetonius Tranquillus (a. AD 69-c. AD I30), Roman imperial administrator and biographer
Calig.
Caligula
De [Illustr.]
De [Illustribus] 
Syme $H O$

Tac.

$\operatorname{TAPh} A$

Tibull.

Val. Max.

Vell. Pat.

Virg.

Vulpe

Watt

Wheeler, 'Topics'

\section{Gramm. Grammaticis}

Div. Aug. Divus Augustus

Div. Jul. Divus Julius

Div. Claud. Divus Claudius

Tib. Tiberius

Vitell. Vitellius

R. Syme, History in Ovid, Oxford 1978

Cornelius Tacitus (c. AD 56-c. r20), Roman historian

$\begin{array}{ll}\text { Ann. } & \text { Annales } \\ \text { Hist. } & \text { Historiae }\end{array}$

Transactions and Proceedings of the American Philological Association

Albius Tibullus (b. c. 50 BC), Roman elegiac poet

Valerius Maximus ( $f$. Ist cent. AD): Roman historian and rhetorician

Velleius Paterculus (c, I9 BC-AD 30/35): Roman soldier and author of Historiae Romanae

P. Vergilius Maro (70-19 BC), Roman pastoral and epic poet

$\begin{array}{ll}\text { Aen. } & \text { Aeneid } \\ \text { Ecl. } & \text { Eclogues } \\ \text { Georg. } & \text { Ceorgics } \\ \text { (Vit. Donat.) } & \text { Vita Donati (the Life of } \\ & \text { Virgil by Aelius } \\ & \text { Donatus) }\end{array}$

R. Vulpe, 'Ovidio nella città dell'esilio', Stud. Ovid., pp. 4I-62

W. S. Watt, 'Notes on Ovid's poems from exile', Illinois Classical Studies 13 (1988), pp. $85-93$

A. L. Wheeler, 'Topics from the life of Ovid', AJPh 46 (r925), pp. I-28 
Wheeler-Goold

Wiedemann

Wilkinson

Witt
A. L. Wheeler, Tristia, Ex Ponto (London, 1924): 2nd ed., rev. G. P. Goold, London/ Cambridge, 1988

T. Wiedemann, 'The political background to Ovid's Tristia II', CQ 25 (1975), pp. 264-7I

L. P. Wilkinson, Ovid Recalled, Cambridge, 1955

R. E. Witt, Isis in the Graeco-Roman

World, London/Ithaca, NY, I97I 